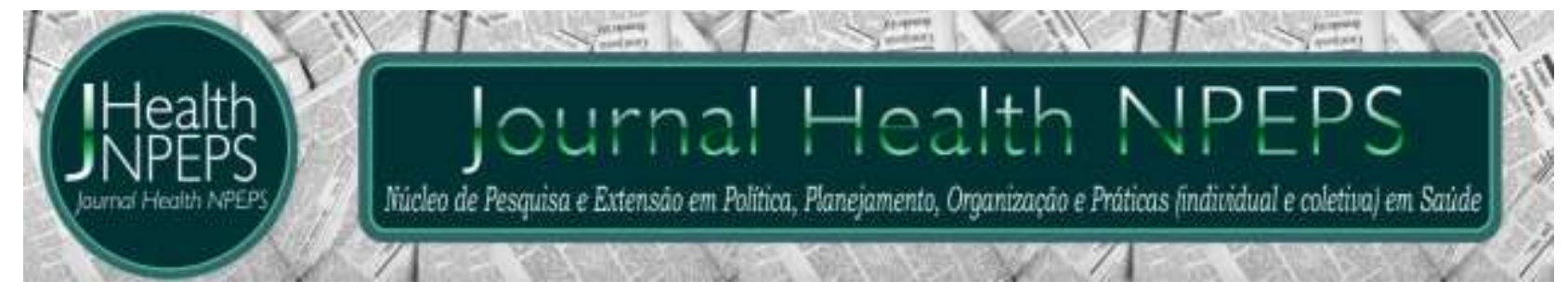

ARTIGO ORIGINAL

\title{
PREVALENCIA DEL USO DEL CINTURÓN DE SEGURIDAD EN VEHÍCULOS AUTOMOTOR: UN ESTUDIO OBSERVACIONAL URBANO
}

\author{
PREVALENCE OF SEATBELT USE IN MOTOR VEHICLES: AN URBAN OBSERVATIONAL \\ STUDY
}

\section{PREVALÊNCIA DO USO DE CINTO DE SEGURANÇA DE VEÍCULOS A MOTOR: UM ESTUDO OBSERVACIONAL URBANO}

\section{Yolanda Velázquez Narváez ${ }^{1}$, Víctor Parra Sierra², José Ignacio Vargas Martínez³ Benito Zamorano González ${ }^{4}$, Fabiola Peña Cárdenas ${ }^{5}$, Lucia Ruiz Ramos ${ }^{6}$, Oscar Monreal Aranda ${ }^{7}$}

\begin{abstract}
RESUMEN
Objetivo: determinar la prevalencia del uso del cinturón de seguridad en conductores y pasajeros de vehículos automotor de la ciudad de $\mathrm{H}$. Matamoros, Tamaulipas. Método: el diseño fue observacional, descriptivo y transversal. La muestra fue de forma aleatoria de 1536 vehículos, se seleccionaron los 6 puntos de mayor tráfico en la ciudad. El instrumento que se utilizó para la recolección de datos fue una lista de verificación por observación para corroborar si los ocupantes del vehículo traían cinturón de seguridad, tomándose los tres primeros vehículos detenidos en fila del carril derecho. El registro se realizó mientras el semáforo se encontraba en la señal de alto total (luz roja). Resultados: se observó una prevalencia del uso del cinturón de seguridad de $37.7 \%$ en conductores de vehículos. El copiloto presento una prevalencia de $12.8 \%$ y los vehículos mayormente utilizados por la muestra fueron auto compacto (42.2\%) y mini van o pick up con 37.7\%. Conclusión: la prevalencia observada en el
\end{abstract}

\footnotetext{
${ }^{1}$ Profesor de tiempo completo, miembro del Cuerpo Académico Psicología, Cognición y cultura. Universidad Autónoma de Tamaulipas, Unidad Académica Multidisciplinaria Matamoros-UAT. México. E-mail: yovelazquez@docentes.uat.edu.mx

2Profesor de tiempo completo, miembro del Cuerpo Académico Psicología y Prevención de Riesgos. Universidad Autónoma de Tamaulipas, Unidad Académica Multidisciplinaria Matamoros-UAT. México. E-mail: vparra@docentes.uat.edu.mx

3Profesor de Tiempo Completo. Cuerpo Académico Piscología y Prevención de Riesgo. Universidad Autónoma de Tamaulipas. Unidad Académica Multidisciplinaria Matamoros-UAT. México. E-mail: jvargas@docentes.uat.edu.mx Autor principal - Dirección de envío: Av. Del Maestro S/N, Colonia Alianza C. P. 87410, Tamps., México.

${ }^{4}$ Profesor de tiempo completo, miembro del Cuerpo Académico Psicología y Prevención de Riesgos. Universidad Autónoma de Tamaulipas, Unidad Académica Multidisciplinaria Matamoros-UAT. México. E-mail: Bzamorano@docentes.uat.edu.mx

${ }^{5}$ Profesor de tiempo completo, miembro del Cuerpo Académico Psicología, Cognición y cultura, Miembro del Sistema Nacional de Investigadores Nivel: Candidato. Universidad Autónoma de Tamaulipas, Unidad Académica Multidisciplinaria Matamoros-UAT. México. E-mail: fcardenas@docentes.uat.edu.mx

${ }^{6}$ Profesor de tiempo completo, miembro del Cuerpo Académico Psicología, Cognición y cultura, Miembro del Sistema Nacional de Investigadores Nivel: Candidato. Universidad Autónoma de Tamaulipas, Unidad Académica Multidisciplinaria Matamoros-UAT. México. E-mail: luruiz@docentes.uat.edu.mx

7 Profesor de tiempo completo, miembro del Cuerpo Académico Psicología y Prevención de Riesgos. Universidad Autónoma de Tamaulipas, Unidad Académica Multidisciplinaria Matamoros-UAT. México. E-mail:
} oscarmonreal1@hotmail.com 
estudio es significativamente más baja para el uso del cinturón de seguridad en comparación con estudios de otros países.

Descriptores: Prevalencia; Equipos de seguridad; Suposición de riesgos.

\section{ABSTRACT}

Objective: to determine the prevalence of seat belt use in drivers and passengers of motor vehicles in the city of $\mathrm{H}$. Matamoros, Tamaulipas. Method: the design was observational cross-sectional descriptive, the sample was randomly 1536 vehicles, 6 points of highest traffic in the city were selected, the instrument that used for data collection was a observation checklist to corroborate If the occupants of the vehicle were wearing a seatbelt, taking the first three vehicles stopped in a row in the right lane, the registration was made while the traffic light was on stop (red light). Results: a prevalence of seat belt use was $37.7 \%$ in vehicle drivers; The co-pilot had a prevalence of $12.8 \%$ and the vehicles mostly used by the sample were autocompact (42.2\%) and mini van or pick up with $37.7 \%$. Conclusion: prevalence for use of seatbelt observed in the study is significantly lower than studies in other countries.

Descriptors: Prevalence; Protective Devices; Risk - Taking.

\section{RESUMO}

Objetivo: determinar a prevalência do uso do cinto de segurança em condutores e passageiros de veículos motorizados na cidade de H. Matamoros, Tamaulipas. Método: o desenho foi observacional, descritivo e transversal. A amostra foi aleatória de 1536 veículos, selecionando em 6 pontos de maior tráfego na cidade. 0 instrumento utilizado pra a coleta de dados foi uma lista de verificação de observação para identificar se os ocupantes do veículo estavam usando cinto de segurança, levando em consideração os três primeiros veículos parados em uma fileira na faixa da direita. 0 registro foi feito enquanto o semáforo estava fechado (luz vermelha). Resultados: a prevalência do uso do cinto de segurança foi de $37,7 \%$ nos motoristas de veículos; 0 co-piloto teve uma prevalência de $12.8 \%$ e os veículos mais utilizados pela amostra foram auto compactos (42.2\%) e mini van ou pick up com $37.7 \%$. Conclusão: a prevalência de uso de cinto de segurança observada no estudo é significativamente menor do que em outros países.

Descritores: Prevalência; Equipamento de Proteção; Assunção de Riscos.

\section{INTRODUCCIÓN}

El progreso continuo de la sociedad moderna ha sido de una manera vertiginosa a partir del siglo XX, donde los avances tecnológicos han permitido mejorar el estilo de vida de la población. Uno de estos avances que ha transformado la vida cotidiana desde su invención hasta la actualidad son los vehículos de motor; Los cuales han contribuido de manera importante al desarrollo de áreas como el transporte, comunicación y desplazamiento de productos y personas.

No obstante los beneficios que han generado existen también aspectos negativos. Uno de los más importantes son los daños ocasionados a la salud, estos los producen los accidentes de tránsito que en el entorno mundial ocasionan alrededor de Journal Health NPEPS. 2017; 2(1):194-205. 
1.24 millones de defunciones, en el continente americano estas defunciones son de 150 mil anualmente, donde también son más de 5 millones de personas que sufren lesiones, lo que equivale 33 lesionados por cada persona fallecida, donde $10 \%$ de estas personas lesionadas sufren alguna incapacidad motriz o sensorial durante todo su vida'

En muchos países la adopción y aplicación de códigos de circulación parecen insuficientes para mitigar el efecto negativo a la salud. La elaboración y observancia eficaz de la legislación son fundamentales para reducir la conducción bajo los efectos del alcohol y el exceso de velocidad y para aumentar el uso de cascos, cinturones de seguridad y cinturones para retención de niños. El informe sobre la situación mundial de la seguridad vial puso de manifiesto que menos de la mitad de los países disponen de leyes para corregir estos 5 factores de riesgo, mientras que solamente el 15\% tiene una legislación que puede considerarse integral en cuanto a su alcance. Tan solo el $57 \%$ de los países dispone la obligatoriedad de usar el cinturón de seguridad para todos los pasajeros, tanto del asiento delantero del vehículo como del trasero ${ }^{1}$.

La OMS encontró que los traumatismos por accidente de tránsito son sistemáticamente una de las tres principales causas de mortalidad en las personas de 15 a 44 años de edad. Además de la muerte, los accidentes en las vías de transito causan muchos traumatismos de menor gravedad: se estima que en el mundo se producen entre 20 y 50 millones de traumatismos no mortales por año².

En el 2004 la OMS y el banco mundial presentaron en forma conjunta el informe mundial sobre prevención de los traumatismos causados por el tránsito, el cual promovió un enfoque integral de la seguridad vial que supone identificar las interacciones entre los usuarios de las vías de tránsito, el vehículo y el entorno vial, las cuales conforman las áreas de intervención. En abril del 2004 después de la presentación del informe mundial la Asamblea General de las Naciones Unidas adopto la resolución 58/289 referente al mejoramiento de la seguridad vial en el mundo y en el mes de marzo del mismo año la asamblea mundial de la salud adopto la resolución WHA 57.10 sobre seguridad vial y salud, la cual instaba a los estados miembros de la OMS a priorizar la seguridad vial como una cuestión de salud pública y llevar a cabo lo necesario para aplicar las medidas para la reducción de traumatismos por accidentes de tránsito ${ }^{3}$.

En él años 2005 y 2008 las resoluciones tomadas por la Asamblea de las Naciones Unidas reforzaron el llamamiento a los estados miembros para aplicar las 
recomendaciones del informe mundial. Entre los factores que disminuyen considerablemente la gravedad de las lesiones causadas por los accidentes de tránsito destaca el uso de dispositivos de retención como el cinturón de seguridad y sillas infantiles para niños y bebes pequeños. Varios estudios han demostrado el efecto protector del cinturón de seguridad al reducir la mortalidad entre un $40 \%$ a $70 \%$ y la gravedad de las lesiones; esta cifra varía en cuestión del tipo de impacto: frontal, lateral o trasero y la posición del pasajero en el vehículo: conductor, pasajero delantero o pasajero trasero ${ }^{3}$.

Un estudio realizado en la Ciudad de Madrid en 1996 sobre la estimación del uso del cinturón de seguridad mediante un estudio de observación y una encuesta telefónica, donde se estudiaron 4067 individuos, de los cuales el 64.6\% fueron hombres y el 35.4\% mujeres. Se obtuvo una prevalencia del uso del cinturón de 58.5\%, con un (IC $95 \% 57,0-60,1)$. La prevalencia para la mujeres fue $61.9 \%$ y para los hombres $56.7 \%$.

En Barcelona, la investigación incluyo a 2442 vehículos privados, comerciales y taxis, arrojo una prevalencia del no uso del cinturón de seguridad de $10.5 \%$ entre los conductores y $4.6 \%$ entre los copilotos, la prevalencia fue superior en los conductores que en los pasajeros, independientemente el uso del vehículo. La prevalencia de los conductores que no utilizan el cinturón de seguridad observada en el estudio es menor a lo reportada en los estudios realizados en Italia e Inglaterra en los cuales también se utilizó la observación directa (24.3\% y $15.3 \%$ respectivamente) $)^{5}$.

De acuerdo con el estudio observacional sobre el cinturón de seguridad y uso de teléfonos móviles entre conductores de vehículos en la ciudad de Doha, Qatar, con una muestra de 2011 vehículos, encontraron que 1463 (72.7\%) de los conductores usaron el cinturón de seguridad (95\% Cl: 70.8 - 74.7\%). La prevalencia del uso del cinturón de seguridad en Doja es menor a otros estudios observacionales reportados como en Michigan, US 94.7\%; en Irán 79.9\%; Cuernavaca, México 72.5\%; Kuwait 55.5\%; Emiratos Árabes Unidos 29\%; Cairo, Egipto 16\% y Lipetskaya, región en Rusia 55\% ${ }^{6}$.

En el estudio avalado por el departamento de transporte tanto de Escocia e Inglaterra ofrece estadísticas sobre la proporción de conductores observados utilizando cinturones de seguridad en el trafico estacionario con una prevalencia del $98.2 \%$ si utilizan el cinturón de seguridad, comparado con las anteriores encuestas realizadas en el 2009, la taza de uso en Inglaterra aumento en el 2014 en comparación con el 2009 para todos los ocupantes del automóvil, excepto para los ocupantes de los asientos 
trasero donde la tasa disminuyo de $89 \%$ a $87.1 \%$ en 2014 . No fue estadísticamente significativo ${ }^{7}$.

En el año 2008 la Secretaria de Salud de México atreves del Centro Nacional de Prevención de Adicciones (CENAPRA), en coordinación con la Organización Panamericana de la Salud (OPS), puso en marcha la iniciativa mexicana de seguridad vial y prevención de lesiones en el tránsito (IMESEVI), la cual implemento estrategias para la promoción de la seguridad vial con el objetivo de reducir lesiones y muertes causadas por el transito; una de las áreas en las que el proyecto esperaba impactar en mayor grado fue el uso del cinturón de seguridad y las silla infantiles. El proyecto fue implementado en las ciudades de León, estado de Guanajuato, Guadalajara (Jalisco), Monterrey (Nuevo León), y ciudad de México, las cuales fueron seleccionadas por su alto índice de siniestralidad vial ${ }^{8}$.

Los resultados muestran que la probabilidad global de usar un dispositivo de retención (cinturón de seguridad o silla infantil) es alrededor del 50\%, el uso de estos dispositivos es más común en la capital Mexicana con una probabilidad del 0.70 , que en el interior de la republica con una 0.35. El mismo estudio encontró como el factor más influyente en el uso del cinturón de seguridad el lugar que el ocupante tiene dentro del vehículo, la mayor parte de los ocupantes se pone el cinturón de seguridad (con probabilidades mayores a 0.80 ) son los conductores, los pasajeros que viajan en la parte trasera del vehículo no tienen el habito de utilizarlo solo un $10 \%$ de los $\operatorname{casos}^{8}$.

En junio del 2011 se publicó en el Diario Oficial de la Federación el acuerdo que se da a conocer la estrategia nacional de seguridad vial 2011-2020, la cual tiene como objetivo general reducir en un $50 \%$ las muertes, así, como reducir al máximo las lesiones y discapacidades por accidentes de tránsito de nuestro país. Los factores sobre cinturón de seguridad que toma en cuenta la estrategia son: legislación y sanciones económicas sobre el uso del cinturón de seguridad, porcentaje de utilización del cinturón de seguridad y porcentaje de defunciones por la no utilización del cinturón de seguridad 9 .

De acuerdo al Instituto Nacional de Geografía y Estadística de México (INEGI, 2015), los accidentes de tránsito terrestre en zonas urbanas y suburbanas en el año 2010 se presentaron un total de 427,267 de accidentes de los cuales los conductores que usaban cinturón de seguridad eran un $25.1 \%$ y los que no lo usaban eran $25.4 \%$ y se ignora si llevaban o no cinturón son 49.5\%, en el 2012 del total de accidentes 390,411 de 
los cuales si llevaban el cinturón $23.8 \%$ y de los que no llevaban cinturón fue de $24.8 \%$ y de los que se desconoce si lo estaban usando o no 51.3\%, en el 2015 del total de accidentes 378,232 , de los que si usaron $20.3 \%$, los que no usaban es de $19.6 \%$ y de los que se ignora el uso o no del cinturón de seguridad $59.9 \%{ }^{10}$.

En el estado de Tamaulipas en el año 2010 se reportaron un total de 13,082 accidentes en la zona urbana y suburbana de los cuales utilizaban el cinturón de seguridad el $25 \%$, los que no usaban eran de $5.5 \%$ y un $69.3 \%$ se desconoce si lo usaban o no, en el 2012 eran 12,808 de los cuales usaban cinturón eran 10.5\%, de los que no usaron fueron 7.3\% y de los que se ignora el uso del cinturón 82.1\%; en el 2015 son 14, 385 accidentes de los cuales el $9.1 \%$ usaban el cinturón de seguridad, de los que no lo usaron el $6.2 \%$ y de los que se desconoce el uso o no es de $84.5 \%{ }^{10}$.

En México, los datos fiables del uso del cinturón de seguridad son escasos. Hasta donde sabemos no se han realizado estudios sistemáticos y a gran escala basados en observaciones directas. El INEGI ha mantenido y extendido desde 1997 una base de datos que proporciona información sobre accidentes de tránsito en zonas urbanas y suburbanas en todo el país, e incluye algunas variables de las características del conductor y condiciones bajo las cuales ocurrió el accidente. Los datos no incluyen la información sobre los pasajeros distintos al conductor, además la información no permite estimar el uso del cinturón de seguridad en la población total, debido a que las personas involucradas en dichos accidentes no forman una muestra aleatoria de la población de usuarios de la vía pública ${ }^{\text {. }}$.

Dichos antecedentes permiten establecer como objetivo principal de la investigación, determinar la prevalencia del uso del cinturón de seguridad en conductores y pasajeros de vehículos de automotor de la ciudad de H. Matamoros, Tamaulipas. El estudio forma parte de las actividades desarrolladas por el grupo de investigación formados por los cuerpos académicos "Psicología, cognición y cultura" y Piscología y prevención de riesgos", correspondiente al proyecto "Factores de riesgo asociados a la siniestralidad vial".

\section{MÉTODO}

Se llevó a cabo un estudio de tipo observacional, descriptivo y transversal sobre la prevalencia del uso del cinturón de seguridad entre los ocupantes de vehículos 
automotores de la ciudad de H. Matamoros, Tamaulipas, México. La muestra ocurrió en forma aleatoria de 1536 vehículos, para el cálculo de la muestra se utilizó la formula $n=\left(Z_{\alpha} p q / e\right)^{2}$, suponiendo un nivel de confianza del $95 \%$, donde $Z_{\alpha}$ es igual a 1.96 y el $\alpha$ 0.05 , y una precisión del $2.5 \%$, e es igual a 0.025 y la peor probabilidad posible $p$ es igual a $0.5^{11}$.

Las observaciones se llevaron a cabo en seis cruces de mayor tráfico vehicular, regulados por semáforos de la ciudad. Los puntos de observación se seleccionaron de acuerdo a los resultados obtenidos del estudio "Contaminación acústica por tránsito vehicular en la frontera de México" realizada por Zamorano-González, Peña-Cárdenas, Parra-Sierra, Velazquez-Narvaez, Vargas-Martínez, Ruiz-Ramos et al ${ }^{12}$, se seleccionaron los 6 puntos de mayor tráfico en la ciudad. Siendo el cruce con mayor afluencia vehicular el de la calle Diagonal Cuauhtémoc y Av. Manuel Cavazos Lerma con 2545 vehículos por hora (Veh/h), seguido del cruce de calle sexta y diagonal Cuauhtémoc con $2484 \mathrm{Veh} / \mathrm{h}$, calle sexta y Av. Manuel Cavazos Lerma con $2422 \mathrm{Veh} / \mathrm{h}$, Av. Laura Villar y Av. Manuel Cavazos Lerma con 2318 Veh/h, Av. Marte R. Gómez y Pedro Cárdenas con 2193 veh/h, Av. y Virgilio Garza y Av. Manuel Cavazos Lerma con 2091 Veh/h.

Para cubrir el total de vehículos de la muestra se consideraron 256 observaciones por cada uno de las cruces, dichas las cuales se llevaron a cabo en las horas de mayor tránsito de dichos cruces. Para este estudio se excluyeron vehículos automotores de dos ruedas.

Seis observadores capacitados con anterioridad para llevar a cabo la realización de las observaciones se dividieron en tres grupos por parejas siéndoles asignados a cada uno dos cruces distintos, la recolección de la información fue aleatoria y sistemática en cada uno de los cruces con semáforo, tomándose los tres primeros vehículos detenidos en fila del carril derecho, el registro se realizó mientras el semáforo se encontraba en la señal de alto total (luz roja) y se registró al conductor y en caso de llevar al copiloto. El instrumento que se utilizó para la recolección de datos fue una lista de verificación por observación para corroborar si los ocupantes del vehículo traían cinturón de seguridad.

El análisis de los datos se llevó a cabo por el paquete estadístico SPSS versión 20, el cálculo de la prevalencia del cinturón de seguridad fue en porcentajes, con un intervalo de confianza (IC) $95 \%$. 


\section{RESULTADOS}

Los resultados presentan las prevalencias del género, tipo de vehículo, el uso del cinturón del pasajero y copiloto, además de la prevalencia del uso del cinturón por género.

Tabla 1. Genero del conductor.

\begin{tabular}{ccc}
\hline Genero & $f$ & $\%$ \\
Masculino & 1063 & 69.3 \\
Femenino & 471 & 30.7 \\
\hline
\end{tabular}

Nota: $f=$ frecuencia $\quad n=1534$

En la tabla 1 observamos que el género masculino (69.3\%) es el que predomina más en relación con el género femenino (30.7\%), para la conducción de los vehículos de automotor que circulan en los puntos seleccionados que correspondes a los de mayor tráfico de la ciudad.

Tabla 2. Tipo de vehículo.

\begin{tabular}{ccc}
\hline Vehículo & $f$ & $\%$ \\
Auto compacto & 646 & 42.2 \\
Mini van o pick up & 577 & 37.7 \\
Transporte publico & 216 & 14.1 \\
Transporte de carga & 66 & 4.3 \\
Vehículo oficial & 25 & 1.6 \\
\hline Nota: $f=$ frecuencia $n=1534$ & &
\end{tabular}

Los tipos de vehículo que más se observaron (tabla 2) durante la realización de las observaciones son: con un $42.2 \%$ los autos compactos, mini van o pick up con $37.7 \%$, seguido del transporte público con $14.1 \%$.

Tabla 3. Prevalencia del uso del cinturón de seguridad del conductor.

\begin{tabular}{ccc}
\hline & $f$ & $\%$ \\
\cline { 2 - 3 } No & 579 & 37.7 \\
No & 955 & 62.3 \\
\hline
\end{tabular}

Nota: $f=$ frecuencia $\quad n=1534$

La tabla 3 se observa que en el $37.7 \%$ prevalece el uso del cinturón de seguridad por parte del conductor contra un $62.3 \%$ no lo utiliza.

Tabla 4. Uso del cinturón de seguridad del copiloto.

\begin{tabular}{ccc}
\hline & $f$ & $\%$ \\
\cline { 2 - 3 } No & 197 & 12.8 \\
No aplica & 486 & 31.7 \\
& 851 & 55.5 \\
\hline
\end{tabular}

Nota: $f=$ frecuencia $\quad n=1534$ 
Lo correspondiente al copiloto la prevalencia del uso del cinturón de seguridad es del $12.8 \%$, los que no lo utilizan son el $31.7 \%$ y el 55.5 de los vehículos de automotor no traían al copiloto.

\section{DISCUSIÓN}

De acuerdo a los datos obtenidos de la investigación se observa que los referente a la prevalencia de género: el género masculino fue el que presento mayor frecuencia con 69.3\%, que concuerda con los encontrado con Godoy, Castel, Peiro, Herrera, Ibañez y Rullan, (1996) los cuales utilizaron el método de observación muy similar encontraron que el $64.6 \%$ de los conductores pertenecían al género masculino. Así, mismo en un estudio relacionado en el año 2014 se encontró una prevalencia de género de conductores masculinos de $70.5 \%$ idéntico a lo obtenido por nuestro estudio de acuerdo a Martínez-Sánchez, Curto, Fu, Martínez, Sureda, Ballbé y Fernández.

En referencia a la prevalencia del uso del cinturón de seguridad, $37.7 \%$ de los conductores del presente estudio lo utilizaron; en el estudio realizado en Barcelona, España ${ }^{5}$ se encontró una prevalencia del uso del cinturón de seguridad del 89.5\%; en otro estudio en Inglaterra y Escocia, llevado a cabo por el Departamento del Transporte del Reino Unido, (2014), encontró una prevalencia del 98.2\% sobre el uso del cinturón de seguridad en los conductores, así, mismo en la ciudad de Doja, Qatar en el año 2015 encontraron una prevalencia del $72.7 \%$, en Madrid, España los resultados de su investigación arrojaron una prevalencia de $58.5 \%{ }^{4}$. En diferentes estudios observacionales reportaron diferentes prevalencias: en Michigan, US. 94.7\%, en Irán 79.9\%, Cuernavaca, México 72.5\%, Kuwait 55.5\%, Lipetskaya, región en Rusia 55\%. Los resultados descritos en los estudios anteriores difieren significativamente de lo encontrado en el presente estudio 6.

En la misma línea de los resultados encontrados en este estudio se encuentran los obtenidos por el INEGI (2015) que reporta una prevalencia del uso del cinturón de seguridad a nivel nacional de 25.1\% en el año 2010, en el año 2012 de $23.8 \%$ y 20.3\% en el año 20015. En lo referente al estado de Tamaulipas reporto una prevalencia de 25\% en el año 2010, 10.5\% en el año 2012 y de 9.1\% en el año 201510; así, como otros estudios reportados en Emiratos Árabes Unidos 29\%, Cairo, Egipto 16\% ${ }^{6}$; las estadísticas reportadas por el INEGI no corresponden a un estudio observacional, si no estadístico en 
base a los accidentes viales reportados en los años correspondientes, donde las prevalencias sobre el uso del cinturón de seguridad son muy similares.

En referencia a la prevalencia del uso del cinturón de seguridad que corresponde al copiloto el presente estudio encontró un valor de $12.8 \%$, contrastando con la prevalencia encontrada por el estudio realizado en Barcelona, España en el 2014 la cual fue de $95.4 \%$.

\section{CONCLUSIÓN}

Se recomienda realizar más estudios observacionales para este fenómeno que causa muchas defunciones y lesiones de gravedad, además las estadísticas con las que cuenta las instituciones gubernamentales son muy escasas porque solamente toman en cuenta los reportes de accidentes de vehículos generados por las autoridades viales los cuales en el mayor de los casos están incompletos y carecen de información acerca del uso de cinturón de seguridad por los ocupantes del vehículo; el instrumento utilizado por las autoridades viales para reportar los datos no se encuentra estandarizado por lo que varía de estado a estado y de municipio a municipio.

En México los estudios de tipo observacional por parte de las autoridades son prácticamente inexistentes, por lo que es importante llevar a cabo este tipo de estudios a un escala mayor y que pueda ser representativa de la población a nivel nacional y se llevan a cabo una mayor difusión, y que los resultados sirvan para tomar medidas que ayuden a la concientización de la importancia del uso del cinturón de seguridad.

\section{REFERENCIAS}

1. OPAS. Informe sobre la situación de la seguridad vial en la región de las Américas 2015. Washington: OPAS; 2015.

2. OMS. Lesiones causadas por el tránsito. 2016 [acceso 20 de febrero 2017]. disponible en: http://www.who.int/mediacentre/factsheets/fs358/es/

3. OMS. Cinturones de seguridad y sistemas de retención infantil: un manual de seguridad vial para decisores y profesionales. Londres: FIA Foundation for the automobile and society; 2009. 
4. Godoy P, Castell J, Peiró E, Herrera D, Ibáñez C, Rullán J. Estimación del uso del cinturón de seguridad mediante un estudio de observación y una encuesta telefónica. Gac Sanit. 1997; 11(1):3-8.

5. Martínez JM, Curto A, Fu M, Martínez C, Sureda X, Ballbé M, Fernández E. Safety belt and mobile pone usage in vehicles in Barcelona (Spain). Gac Sanit. 2014; 28(4):305-308.

6. Mahfoud ZR, Cheema S, Alroh H, Al-Thani MH, Al-Thani AAM, Mamtani R. Seat belt and mobile phone use among vehicle drivers in the city of Doha, Qatar: and Observational Study. BMC Public Health. 2015; 15:937.

7. Department for transport. Seat belt and mobile phone use surveys: England and Scotland, 2014. Transport Scotland and Department for transport. 2015 [acceso 27 de febrero 2017] disponible en: https://www.gov.uk/government/uploads/system/uploads/attachment_data/file/ 406723/seatbelt-and-mobile-use-surveys-2014.pdf

8. Cervantes A, Rojas R, Leenen I, Givaudan M, García G, Palomares G. IMESEVI, Iniciativa mexicana de seguridad vial, metodología para la medición d cuatro factores de riesgo en materia de seguridad vial. México: Secretaria de Salud; 2011.

9. Observatorio vial. Indicadores estatales de seguridad vial. Observatorio vial por una cultura y convivencia vial. 2013 [acceso 27 de febrero 2017]. Disponible en: www.observatoriovial.com/publicaciones/publicaciones/docs/Indicadores_Estado_ de_Mexico.PDF

10. Instituto Nacional de Estadística y Geografía. Accidentes de tránsito terrestres en zonas urbanas y suburbanas. 2015 [acceso 27 de febrero 2017]. Disponible en: http://www.inegi.org.mx/sistemas/olap/Proyectos/bd/continuas/transporte/accid entes.asp?s=est\&c=13159\&proy=atus_accidentes

11. Fernández P. Determinación del tamaño muestral. Cad Aten Primaria. 2001; 3:13814.

12. Zamorano B, Peña F, Parra V, Velázquez Y, Vargas JI, Ruiz L, Monreal 0. Contaminación acústica por tránsito vehicular en la frontera de México. Congreso internacional de ciencias básicas e ingenierías-CICI - Villavicencio, Colombia. 2016 [acceso 27 de febrero 2017]. Disponible en: http://fcbi.unillanos.edu.co/cici/Articulos/CICI_2016_paper_59.pdf 
Conflito de interesses: Os autores declaram não haver conflito de interesses.

Como citar este artigo: Narváez YV, Sierra VP, Martínez JIV, González BZ, Cárdenas FP, Ramos LR, et al. Prevalencia del uso del cinturón de seguridad en vehículos automotor: un estudio observacional urbano. Journal Health NPEPS. 2017; 2(1):194-205. 